\title{
Anticoagulación en paciente con trombocitopenia secundaria a Síndrome Antifosfolípido: Reporte de Caso
}

\author{
Laura C. Pedraza ${ }^{1 *}$, Yeferson A. Fajardo², Renato A. Guzmán ${ }^{3}$
}

\begin{abstract}
${ }^{1}$ Médico Interno Investigación, Facultad de Medicina, Fundación Universitaria Juan N. Corpas, Bogotá D.C., Bogotá. (Colombia). ${ }^{2}$ Médico Epidemiólogo. Residente de Especialización Medicina Familiar Integral. Fundación Universitaria Juan N. Corpas. Bogotá. (Colombia). ${ }^{3}$ Médico Internista, Especialista en Inmunología y Reumatología, Instituto de Enfermedades Autoinmunes Renato Guzmán, Bogotá D.C., Colombia. Profesor Titular de Inmunología y Medicina Interna. Departamento de Medicina Interna, Fundación Universitaria Juan N. Corpas, Clínica Corpas. Bogotá. (Colombia).
\end{abstract}

\section{RESUMEN}

Introducción. En el contexto de un síndrome antifosfolípido primario (SAF), la trombocitopenia, paradójicamente, no sugiere riesgo de sangrado sino de trombosis, lo que podría evolucionar a un SAF catastrófico, una patología con mortalidad elevada, por ello en casos de SAF con trombocitopenia la anticoagulación no debe diferirse, sino que es el manejo inmediato. A continuación, se describe un caso de SAF en un hombre de 20 años con epistaxis, hemorragia gingival, disnea, hemoptisis y equimosis en miembro inferior izquierdo, con antecedente de tromboembolismo pulmonar (TEP) secundario a trombosis venosa profunda (TVP) hace 2 años, por lo que estaba anticoagulado con cumarínico, pero no lo pudo continuar desde hace 6 meses por trámites administrativos. Se considera posible TEP por lo que se inicia enoxaparina y se solicitan paraclínicos para aclarar etiología. El Angiotac confirma el diagnóstico y el Doppler de miembros inferiores descarta TVP. Además, se confirma un SAF primario y se empieza a notar una trombocitopenia marcada que no es la esperada con esta patología, por lo que se discute si anticoagular o no, sopesando riesgo- beneficio de lo anterior. Adicionalmente, dada la etiología autoinmune, se inicia manejo con metilprednisolona, azatioprina e hidroxicloroquina, con mejoría clínica y paraclínica, lográndose terapia puente para cumarínico satisfactoriamente, por lo que fue posible dar egreso con Warfarina, corticoesteroide oral, azatioprina $\mathrm{e}$ hidroxicloroquina. Basados en el caso expuesto, afirmamos que los pacientes con SAF y trombocitopenia deben ser anticoagulados tan pronto como sea posible.

Palabras clave: síndrome antifosfolípido primario, anticoagulación, trombocitopenia.

\section{ABSTRACT}

Anticoagulation in a patient with thrombocytopenia Secondary to antiphospholipid syndrome: Case Report

Introduction. In the context of primary antiphospholipid syndrome (APS), thrombocytopenia, paradoxically, doesn't suggest bleeding but thrombosis and it can progress to a catastrophic APS, a disease with elevated mortality. For that, in cases of APS with thrombocytopenia, the anticoagulation should not be differed, instead is the immediate treatment. Next is described a case of APS in a 20-year-old man with epistaxis, gingival bleeding, dyspnea, hemoptysis and ecchymosis in left inferior limb, with history of pulmonary thromboembolism (PTE) secondary to DVT 2 years ago, so he was anticoagulated with coumarin that was stopped 6 months ago due to administrative procedures. A PTE was considered so enoxaparin is started, and tests were ordered to clarify the etiology. The angiotac confirms the diagnosis and inferior limbs Doppler rules out DVT. Furthermore, a primary APS is confirmed and there is noted a severe thrombocytopenia unexpected for this pathology, that is why anticoagulation is discussed, weighing risk- benefit. In addition, due to autoimmune etiology, methylprednisolone, azathioprine and hydroxychloroquine was started with clinical and paraclinical improvement, achieving bridge therapy to coumarin satisfactorily, being possible to discharge the patient with warfarin, oral corticosteroid, azathioprine and hydroxychloroquine. Based on the exposed case, we affirm that patients with APS and thrombocytopenia must be anticoagulated as soon as possible.

Keywords: primary antiphospholipid syndrome, anticoagulation, thrombocytopenia.

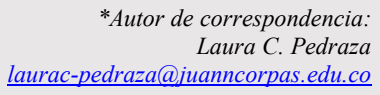

Como citar: Pedraza, LC, Fajardo, AY, Guzmán, RA, Anticoagulación en paciente con trombocitopenia secundaria a Sindrome Antifosfolipido: Reporte de Caso?. Revista Cuarzo 2019;25(2):34-37.

Recibido: 15 de julio de 2019 Aceptado: 20 de septiembre de 2019 Publicado: 30 de diciembre de 2019 DOI: https://doi.org/10.26752/cuarzo.v25.n2.445 


\section{INTRODUCCIÓN}

E $1 \mathrm{SAF}$ en una enfermedad caracterizada por trombosis recurrentes, morbilidad materna y la presencia de al menos un anticuerpo antifosfolípido positivo (1). Puede ser primario o secundario, en un 50\% de los casos, y la entidad a la que más frecuentemente se encuentra asociado es a lupus eritematoso sistémico (LES). Su prevalencia es de 50/100.000 y su incidencia anual de 2.1 en Estados Unidos (2).

En cuanto al diagnóstico, se hace con las manifestaciones clínicas y con exámenes de laboratorio. A continuación, se mencionan las manifestaciones más comunes, en orden descendente: TVP, livedo reticularis, accidente cerebrovascular, tromboflebitis superficial, TEP, aborto y accidente isquémico transitorio (3). Otras manifestaciones menos comunes son la enfermedad valvular, hipertensión pulmonar, úlceras cutáneas, insuficiencia adrenal, déficit cognitivo, amaurosis fugaz, necrosis de medula ósea y osteonecrosis $(3,4)$. Respecto a los exámenes de laboratorio, con uno de los siguientes positivos junto con las manifestaciones clínicas se realiza el diagnóstico: anticuerpos anticardiolipina $\operatorname{IgM}$ o $\operatorname{IgG}$, anticuerpos anti beta 2 glicoproteína I y anticoagulante lúpico $(1,5)$, siendo este último el que más se relaciona con las manifestaciones clínicas (1). Si bien no es un criterio diagnóstico, se puede evidenciar también una disminución en el complemento, así como trombocitopenia (3).

Por otra parte, el tratamiento se basa más en prevención secundaria que en prevención primaria dado que esta última no ha mostrado ser efectiva, excepto en casos muy específicos como en personas con factores de riesgo cardiovascular (tabaquismo, obesidad e hipertensión arterial), en quienes se indicará aspirina a bajas dosis (75-100 mg diaria). Otra indicación para prevención primaria son los pacientes con LES, quienes pueden recibir aspirina a bajas dosis o hidroxicloroquina $200-400 \mathrm{mg}$ al día. En el caso de mujeres en embarazo con abortos recurrentes antes de la semana 10 de gestación, se puede indicar aspirina a bajas dosis preconcepcional o heparina de bajo peso molecular a dosis trombo profiláctica. Si la mujer ha tenido complicaciones en embarazos previos después de la semana 10 de gestación, debe recibir aspirina a bajas dosis preconcepcional y heparina de bajo peso molecular a dosis trombo profiláctica, pero si ya ha tenido episodios trombóticos recibirá tanto aspirina a bajas dosis como heparina de bajo peso molecular a dosis terapéutica. El tratamiento en personas que acuden a urgencias y se les confirma el diagnostico, dependerá si el episodio trombótico es venoso o arterial, pues en el primer caso se indicara warfarina para un INR de 2 a 3, mientras que en el segundo caso se formulara warfarina para INR de 3 a 4 o aspirina a bajas dosis con warfarina para un INR de 2 a 3 (6).

Teniendo en cuenta que el diagnóstico, el tratamiento y el pronóstico del SAF dependen de tener la sospecha de la patología para tratarla rápidamente y evitar complicaciones, a continuación, se presenta un caso clínico de SAF primario en paciente joven con trombosis recurrentes.

\section{PRESENTACIÓN DEL CASO}

Paciente masculino de 20 años quien ingresa al servicio de urgencias por presentar cuadro clínico de 3 semanas de evolución consistente en episodios recurrentes de epistaxis asociado a hemorragia gingival, equimosis en miembro inferior izquierdo que limita la marcha, disnea y hemoptisis. Como antecedente de importancia, el paciente presento TVP hace 2 años por lo que estaba anticoagulado con Warfarina la cual suspendió hace 6 meses por trámites administrativos. Resto de antecedentes negativos. Al ingreso, examen físico con signos vitales normales, como hallazgos positivos, dolor en gastronemio izquierdo, lesiones tipo púrpura en piel sin sangrado activo. Con estos hallazgos, se considera TEP de alta probabilidad según escala de Wells, por lo que se solicita cuadro hemático, tiempos de coagulación, dímero $\mathrm{D}$, radiografía y Angiotac de tórax y Doppler venoso de miembro inferior izquierdo. Por el momento se decide hospitalizar por el servicio de medicina interna e iniciar manejo con enoxaparina subcutánea $50 \mathrm{mg}$ cada 12 horas.

Se recibe reporte de paraclínicos con plaquetas $16.000 \mathrm{u} / \mathrm{L}$ y frotis sanguíneo con macroplaquetas; PT 17.5, INR 1.39, PTT 35 , dímero $\mathrm{D} 0.61$, radiografía de tórax con borramiento del ángulo costofrénico lateral derecho por presencia de escasa cantidad de líquido pleural, Doppler negativo para TVP y pendiente el Angiotac. En el cuadro hemático de ingreso, llama la atención la trombocitopenia además de persistencia de epistaxis y de disnea por lo que deciden suspender anticoagulación y ordenar trasfusión de 6 unidades de plaquetas, así como solicitar paraclínicos para autoinmunidad y discrasia sanguínea durante el primer día de hospitalización. Se encuentran Coomb's directo positivo $2+$, ANAs y ENAs negativos, anticoagulante lúpico positivo; antitrombina III, proteína C y factor VIII normales, con 23.100 plaquetas después de la transfusión.

El segundo día de hospitalización se toma cuadro hemático con aumento en cifras de plaquetas llegando a $24.000 \mathrm{u} / \mathrm{L}$, sin embargo, teniendo en cuenta el anticoagulante lúpico junto con los antecedentes trombóticos, se diagnostica SAF por lo que se interconsulta al servicio de reumatología para optimizar manejo. Además, se recibe reporte de Angiotac que evidencia TEP en resolución y trombo organizado en la cara posterior de la arteria pulmonar derecha ligeramente irregular, de aproximadamente $24 \times 7 \mathrm{~mm}$ que genera una irregularidad de la cara posterior de la luz de la arteria pulmonar derecha, conllevando a una estenosis de la arteria pulmonar, considerando TEP, pero el paciente seguía sin anticoagulación por la trombocitopenia.

Al día siguiente, dada la no mejoría del cuadro, se decide iniciar manejo con metilprednisolona $500 \mathrm{mg}$ en $100 \mathrm{cc}$ de solución salina al día durante 3 días y posterior continuación con corticoesteroide por vía oral, sin embargo, el manejo no se 
aplicó durante los 3 días por lo que, en el cuarto día de hospitalización, cuando el servicio de reumatología valora al paciente, persiste con epistaxis y disnea. Así pues, de una parte se ratifica el diagnóstico de SAF primario dado que no hay manifestaciones ni laboratorios que sugieran otra enfermedad de base como LES, por lo que se considera que la anticoagulación no debe retrasarse más dado que los beneficios sobrepasan los riesgos, además se solicita que no se realicen trasfusiones teniendo en cuenta el origen autoinmune de la patología y por otro lado se reinician pulsos de metilprednisolona $500 \mathrm{mg}$ en $100 \mathrm{cc}$ de solución salina al día durante 3 días y posterior continuación por vía oral con prednisona $10 \mathrm{mg}$ por kilogramo al día. Llamativamente se observa un nuevo descenso en las plaquetas con cifras de $14.000 \mathrm{u} / \mathrm{L}$, atribuido a la transfusión realizada.

Dos días después de terminar el corticoesteroide, el paciente refiere amaurosis fugaz y persistencia de epistaxis diaria, así como persiste trombocitopenia con $11.000 \mathrm{u} / \mathrm{L}$, por lo que se adicionan azatioprina $50 \mathrm{mg}$ cada 12 horas e hidroxicloroquina $200 \mathrm{mg}$ al día considerando que, más que manifestación del $\mathrm{SAF}$, esté relacionada con purpura trombocitopenica idiopática. En la Tabla 1 se puede apreciar el comportamiento de las plaquetas durante la hospitalización, lo que obligo a mantener el paciente en observación hasta notar un ascenso en el día 8 de hospitalización.

El día 11 de hospitalización se realiza terapia puente a Warfarina y 3 días después, con la mejoría clínica y con plaquetas de $50.000 \mathrm{u} / \mathrm{L}$ después de 14 días de hospitalización, se da alta hospitalaria con cita de control por reumatología en 4 semanas y ordenes ambulatorias para continuar Warfarina, corticoesteroide oral, azatioprina e hidroxicloroquina.

Tabla 1: Valores de las Plaquetas y su Relación con Eventos Importantes Ocurridos Durante la Hospitalización del Paciente en Mención.

\begin{tabular}{ccl}
\hline Día & $\begin{array}{c}\text { Plaquetas } \\
\mathbf{u} / \mathbf{L}\end{array}$ & \multicolumn{1}{c}{ Evento importante } \\
\hline $\mathbf{1}$ & 16.000 & $\begin{array}{l}\text { Transfusión de plaquetas } \\
\text { Interconsulta a reumatología por } \\
\text { diagnóstico de SAF }\end{array}$ \\
$\mathbf{2}$ & 24.000 & Día 1 de corticoide intravenoso \\
$\mathbf{4}$ & 14.000 & Día 2 de corticoide intravenoso \\
$\mathbf{5}$ & 16.000 & Día 3 de corticoide intravenoso \\
$\mathbf{6}$ & 11.000 & Día 1 de corticoide oral \\
$\mathbf{8}$ & 23.000 & Día 2 de corticoide oral \\
$\mathbf{1 0}$ & 36.000 & Día 3 de corticoide oral \\
$\mathbf{1 2}$ & 37.000 & Egreso. Manejo ambulatorio \\
$\mathbf{1 3}$ & 50.000 & Fuente: Autores \\
\hline
\end{tabular}

\section{DISCUSIÓN}

El SAF es una enfermedad frecuentemente asociada a patologías autoinmunes, sin embargo, puede presentarse como una entidad primaria y su manejo debe establecerse rápidamente para evitar desenlaces catastróficos. Se expuso el caso de un paciente con trombosis recurrentes quien estaba anticoagulado con Warfarina y la suspendió, se presenta con un cuadro sugestivo de recurrencia trombótica, pero con una trombocitopenia muy llamativa y dado que el SAF suele cursar con valores por encima de los $50.000 \mathrm{u} / \mathrm{L}$ que no requieren tratamiento, se consideró que además, este paciente estuviera cursando simultáneamente con una purpura trombocitopenica idiopática, que puede tener anticuerpos antifosfolípido positivos (7). La trombocitopenia no es una manifestación tan común del SAF y hace parte de las expresiones clínicas que no son criterio para su diagnóstico $(1,4,8)$, resaltando que algunos lo han asociado a LES en un 5 a $8 \%$ con un mecanismo de acción que comprende la activación y el consumo plaquetarios o microangiopatía trombótica (9-11). En la actualidad, no existen esquemas definidos para tratar casos refractarios, pero se pueden utilizar corticoesteroides y rituximab, resaltando que hace algunos años nosotros informamos de la utilidad del rituximab en pacientes con SAF (12).

Respecto a la anticoagulación se puede extrapolar el siguiente esquema que se utiliza en pacientes con cáncer y trombocitopenia: cuando las plaquetas sean mayores a 50.000 $\mathrm{u} / \mathrm{L}$ se administra enoxaparina a dosis plena, si están entre 25.000 y $50.000 \mathrm{u} / \mathrm{L}$ la dosis debe disminuirse a la mitad y si es están por debajo de $20.000 \mathrm{u} / \mathrm{L}$ la sugerencia es no anticoagular (13).

El manejo era fundamental en este caso, pero se presentó un debate al momento del mismo, teniendo en cuenta que la terapia con anticoagulantes se considera segura en pacientes sin sangrado activo con al menos 50.000 plaquetas o incluso menos si ya están recibiendo tratamiento para la trombocitopenia y las cifras no mejoran (14), el paciente no alcanzaba dicho requerimiento y se debió sopesar el riesgo - beneficio de la anticoagulación versus el riesgo de sangrado, si bien se ha documentado que en estos pacientes la trombocitopenia ocasionalmente predice el desarrollo paradójico de eventos trombóticos y SAF catastrófico (15) por lo que se decidió entonces, dar manejo anticoagulante con heparina de bajo peso molecular y para controlar la trombocitopenia, se indicaron pulsos de corticoesteroide junto con azatioprina $(8,14,16,17)$, con una mejoría en las cifras de plaquetas y sin complicaciones hemorrágicas por lo cual, basados en el caso expuesto, en pacientes con SAF y trombocitopenia sugerimos anticoagulación plena e inmunosupresión. Es importante recordar que, por la naturaleza autoinmune de la trombocitopenia, la transfusión no está indicada y de hecho puede agravar del cuadro clínico, por lo que solo es útil en casos muy puntuales (8). También es importante recordar que, estos pacientes deben ser valorados después del alta hospitalaria, pues el diagnostico se debe confirmar con la persistencia de los anticuerpos positivos al menos 12 semanas después debido a que, por ejemplo, el anticoagulante lúpico puede ser un falso positivo en pacientes anticoagulados (1).

El paciente evoluciono favorablemente y se le dio egreso con anticoagulación y corticoesteroide vía oral, así como control médico al mes. 


\section{CONCLUSIONES}

La asociación de la trombocitopenia severa en SAF es infrecuente, pero al presentarse genera controversias en la decisión terapéutica, si bien la instauración temprana y eficaz de terapia anticoagulante es fundamental, y en consideración de trombocitopenia refractaria el uso de pulsos de corticoterapia en inmunomodulación no debe esperarse ante la sospecha de autoinmunidad. Los agonistas de trombopoyetina no tienen mayor valor y aumentan el riesgo de trombosis y debemos recordar que esta condición por esencia es de naturaleza trombofílica. Es un dilema terapéutico grande, cada caso se debe individualizar y prima el criterio clínico para tomar decisiones al respecto.

Conflicto de Interés: Los autores declaran no tener ningún conflicto de interés.

\section{REFERENCIAS}

1. Garcia D, Erkan D. Diagnosis and Management of the Antiphospholipid Syndrome. N Engl J Med. 2018; 378:2010-21.

2. Duarte-García A, Pham MM, Crowson CS, Amin S, et al. The Epidemiology of Antiphospholipid Syndrome: A Population-Based Study. Arthritis Rheumatol. septiembre de 2019;71(9):1545-52.

3. Clinical manifestations of antiphospholipid syndrome UpToDate [Internet]. [citado 21 de octubre de 2019]. Disponible en: https://www-uptodatecom.recursosenlinea.juanncorpas.edu.co:2443/contents/clin ical-manifestations-of-antiphospholipid-

syndrome? search $=\mathrm{Clinical} \% 20$ manifestations $\% 20 \mathrm{of} \% 20$ an tiphospholipid\%20syndrome\&source=search_result\&select edTitle $=1 \sim 150 \&$ usage_type $=$ default\&display_rank $=1$

4. Sciascia S, Amigo M-C, Roccatello D, et al. Diagnosing antiphospholipid syndrome: «extra-criteria» manifestations and technical advances. Nat Rev Rheumatol. septiembre de 2017;13(9):548-60.

5. Diagnosis of antiphospholipid syndrome - UpToDate [Internet]. [citado 21 de octubre de 2019]. Disponible en: https://www-uptodate-

com.recursosenlinea.juanncorpas.edu.co:2443/contents/dia gnosis-of-antiphospholipid-

syndrome?search=Diagnosis $\% 20$ of $\% 20$ antiphospholipid $\%$ 20 syndrome\&source $=$ search_result\&selectedTitle $=1 \sim 150 \&$ usage_type $=$ default\&display_rank $=1$

6. Uthman I, Noureldine MHA, Ruiz-Irastorza G, et al. anagement of antiphospholipid syndrome. Ann Rheum Dis. febrero de 2019;78(2):155-61.

7. Demetrio Pablo R, Munoz P, Lopez-Hoyos M, et al. Thrombocytopenia as a thrombotic risk factor in patients with antiphospholipid antibodies without disease criteria. Med Clin (Barc). 10 de mayo de 2017;148(9):394-400.

8. Antiphospholipid Syndrome and Thrombocytopenia | IntechOpen [Internet]. [citado 21 de octubre de 2019]. Disponible

en: https://www.intechopen.com/books/thrombocytopenia/anti phospholipid-syndrome-and-thrombocytopenia

9. Guzmán Moreno RA. Sindrome Antifosfolipido. Medicas UIS. 2001; 15:123-35.
10. Guzmán Moreno RA. Síndrome Antifosfolípido (Síndrome de Hughes). Rev Colomb Reum. 2001; 8:147-64.

11. Vreede AP, Bockenstedt PL, McCune WJ, et al. Cryptic conspirators: a conversation about thrombocytopenia and antiphospholipid syndrome. Curr Opin Rheumatol. mayo de 2019;31(3):231-40.

12. Guzman RA. B Cell depletion in autoimmune diseases. Advance in autoimmunity. Autoimm Rev 2009; 8:585-90.

13. Mantha S, Miao Y, Wills J, et al. Enoxaparin dose reduction for thrombocytopenia in patients with cancer: a quality assessment study. J Thromb Thrombolysis. mayo de 2017;43(4):514-8.

14. Treatment of antiphospholipid syndrome - UpToDate [Internet]. [citado 21 de octubre de 2019]. Disponible en: https://www-uptodate-

com.recursosenlinea.juanncorpas.edu.co:2443/contents/sear ch?search $=$ Treatment $\% 20$ of $\% 20$ antiphospholipid $\% 20$ syndr ome \&sp $=0 \&$ search Type $=$ PLAIN_TEXT\&source $=$ USER_I NPUT\&searchControl=TOP_PULLDOWN\&searchOffset $=$ $1 \&$ auto Complete $=$ false \&language $=$ es $\& \max =10 \&$ index $=\& a$ utoCompleteTerm=

15. Pontara E, Banzato A, Bison E, et al. Thrombocytopenia in high-risk patients with antiphospholipid syndrome. J Thromb Haemost JTH. marzo de 2018;16(3):529-32.

16. Atsumi T, Furukawa S, Amengual O, et al. Antiphospholipid antibody associated thrombocytopenia and the paradoxical risk of thrombosis. Lupus. 2005;14(7):499-504.

17. Skride A, Sablinskis M, Sablinskis K, et al. Chronic Thromboembolic Pulmonary Hypertension and Antiphospholipid Syndrome with Immune Thrombocytopenia: A Case Report. Am J Case Rep. 19 de octubre de 2018; 19:1245-8. 